\title{
Impact of the Radiation Background on the CMS muon high-eta upgrade for the LHC high luminosity scenario
}

D. Abbaneo ${ }^{15}$, M. Abbas ${ }^{15}$, M. Abbrescia ${ }^{2}$, A.A. Abdelalim ${ }^{8}$, M. Abi Akl ${ }^{13}$, W. Ahmed ${ }^{8}$, W. Ahmed ${ }^{17}$, P. Altieri ${ }^{2}$, R. Aly ${ }^{8}$, A. Ashfaq ${ }^{17}$, P. Aspell ${ }^{15}$, Y. Assran ${ }^{7}$, I. Awan ${ }^{17}$, S. Bally ${ }^{15}$, Y. Ban ${ }^{3}$, S. Banerjee ${ }^{19}$, P. Barria ${ }^{5}$, L. Benussi ${ }^{14}$, V. Bhopatkar ${ }^{22}$, S. Bianco ${ }^{14}$, J. Bos ${ }^{15}$, O. Bouhali ${ }^{13}$, S. Braibant ${ }^{4}$, S. Buontempo ${ }^{24}$, J. Cai ${ }^{3}$, C. Calabria ${ }^{2}$, C. Caputo ${ }^{2}$, F. Cassese ${ }^{24}$, A. Castaneda ${ }^{d *}$, S. Cauwenbergh ${ }^{16}$, F.R. Cavallo ${ }^{4}$, A. Celik ${ }^{9}$, M. Choi ${ }^{31}$, K. Choi ${ }^{31}$, S. Choi ${ }^{29}$, J. Christiansen ${ }^{15}$, A. Cimmino ${ }^{16}$, S. Colafranceschi ${ }^{15}$, A. Colaleo ${ }^{2}$, A. Conde Garcia ${ }^{15}$, M.M. Dabrowski ${ }^{15}$, G. De Lentdecker ${ }^{5}$, R. De Oliveira ${ }^{15}$, G. de Robertis ${ }^{2}$, S. Dildick ${ }^{9,16}$, B. Dorney ${ }^{15}$, W. Elmetenawee ${ }^{8}$, G. Fabrice $^{27}$, S. Ferry ${ }^{15}$, P. Giacomelli ${ }^{4}$, J. Gilmore ${ }^{9}$, L. Guiducci ${ }^{4}$, A. Gutierrez ${ }^{12}$, R.M. Hadjiiska ${ }^{28}$, A. Hassan ${ }^{8}$, J. Hauser ${ }^{21}$, K. Hoepfner ${ }^{1}$, M. Hohlmann ${ }^{22}$, H. Hoorani ${ }^{17}$, Y.G. Jeng ${ }^{18}$, T. Kamon ${ }^{9}$, P.E. Karchin ${ }^{12}$, H. Kim ${ }^{18}$, S. Krutelyov ${ }^{9}$, A. Kumar ${ }^{11}$, J. Lee ${ }^{31}$, J. Lee ${ }^{31}$, T. Lenzi ${ }^{5}$, L. Litov ${ }^{28}$, F. Loddo ${ }^{2}$, T. Maerschalk ${ }^{5}$, G. Magazzu ${ }^{26}$, M. Maggi ${ }^{2}$, Y. Maghrbi ${ }^{13}$, A. Magnani ${ }^{25}$, N. Majumdar ${ }^{19}$, P.K. Mal ${ }^{6}$, K. Mandal ${ }^{6}$, A. Marchioro ${ }^{15}$, A. Marinov ${ }^{15}$, J.A. Merlin ${ }^{15}$, N. Mohammed ${ }^{11}$, A.K. Mohanty ${ }^{23}$, A. Mohapatra ${ }^{22}$, S. Muhammad ${ }^{17}$,

S. Mukhopadhyay ${ }^{19}$, S. Nuzzo ${ }^{2}$, E. Oliveri ${ }^{15}$, L.M. Pant ${ }^{23}$, P. Paolucci ${ }^{24}$, I. Park ${ }^{31}$, G. Passeggio ${ }^{24}$, B. Pavlov ${ }^{28}$, B. Philipps ${ }^{1}$, M. Phipps ${ }^{22}$, D. Piccolo ${ }^{14}$, H. Postema ${ }^{15}$, G. Pugliese ${ }^{2}$, A. Puig Baranac ${ }^{15}$, A. Radi ${ }^{7}$, R. Radogna ${ }^{2}$, G. Raffone ${ }^{14}$, S. Ramkrishna ${ }^{11}$, A. Ranieri ${ }^{2}$, C. Riccardi ${ }^{25}$, A. Rodrigues ${ }^{15}$, L. Ropelewski ${ }^{15}$, S. Roychoddhury ${ }^{19}$, M.S. Ryu ${ }^{18}$, G. Ryu ${ }^{31}$, A. Safonov ${ }^{9}$, A. Sakharov ${ }^{10}$, S. Salva ${ }^{16}$, G. Saviano ${ }^{14}$, A. Sharma ${ }^{15}$, S.K. Swain ${ }^{6}$, J.P. Talvitie ${ }^{15,20}$, C. Tamma ${ }^{2}$, A. Tatarinov ${ }^{9}$, N. Turini ${ }^{26}$, T. Tuuva ${ }^{20}$, J. Twigger ${ }^{22}$, M. Tytgat $^{16}$, I. Vai ${ }^{25}$, M. van Stenis ${ }^{15}$, R. Venditi ${ }^{2}$, E. Verhagen ${ }^{5}$, P. Verwilligen ${ }^{2}$, P. Vitulo ${ }^{25}$, U. Yang ${ }^{30}$, Y. Yang $^{5}$, R. Yonamine ${ }^{5}$, N. Zaganidis ${ }^{16}$, F. Zenoni ${ }^{5}$, A. Zhang ${ }^{22}$ 
${ }^{1}$ RWTH Aachen University, III Physikalisches Institut A, Aachen, Germany ${ }^{2}$ Politecnico di Bari, Universitá di Bari and INFN Sezione di Bari, Bari, Italy

${ }^{3}$ Peking University, Beijing, China

${ }^{4}$ University and INFN Bologna, Bologna, Italy

${ }^{5}$ Université Libre de Bruxelles, Brussels, Belgium

${ }^{6}$ National Institute of Science Education and Research, Bhubaneswar, India

${ }^{7}$ Academy of Scientific Research and Technology, ENHEP, Cairo, Egypt

${ }^{8}$ Helwan University \& CTP, Cairo, Egypt

${ }^{9}$ Texas A\&M University, College Station, USA

${ }^{10}$ Kyungpook National University, Daegu, Korea

${ }^{11}$ University of Delhi, Delhi, India

${ }^{12}$ Wayne State University, Detroit, USA

${ }^{13}$ Texas A\&M University at Qatar, Doha, Qatar

${ }^{14}$ Laboratori Nazionali di Frascati - INFN, Frascati, Italy

${ }^{15}$ CERN, Geneva, Switzerland

${ }^{16}$ Ghent University, Dept. of Physics and Astronomy, Ghent, Belgium

${ }^{17}$ National Center for Physics, Quaid-i-Azam University Campus, Islamabad, Pakistan

${ }^{18}$ Chonbuk National University, Jeonju, Korea

${ }^{19}$ Saha Institute of Nuclear Physics, Kolkata, India

${ }^{20}$ Lappeenranta University of Technology, Lappeenranta, Finland

${ }^{21}$ University of California, Los Angeles, USA

${ }^{22}$ Florida Institute of Technology, Melbourne, USA

${ }^{23}$ Bhabha Atomic Research Centre, Mumbai, India

${ }^{24}$ INFN Napoli, Napoli, Italy

${ }^{25}$ INFN Pavia and University of Pavia, Pavia, Italy

${ }^{26}$ INFN Sezione di Pisa, Pisa, Italy

${ }^{27}$ IRFU CEA-Saclay, Saclay, France

${ }^{28}$ Sofia University, Sofia, Bulgaria

${ }^{29}$ Korea University, Seoul, Korea

${ }^{30}$ Seoul National University, Seoul, Korea

${ }^{31}$ University of Seoul, Seoul, Korea

E-mail: castanedecern.ch 


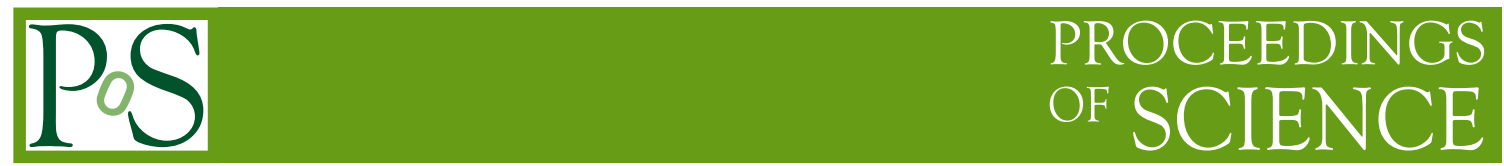

The Compact Muon Solenoid (CMS) experiment at the LHC is planning an upgrade of its muon detection system aiming to extend the muon detection capabilities in the forward region with the installation of new muon stations based on Gas Electron Multiplier (GEM) and Resistive Plate Chambers (RPC) technologies during the so-called Phase-2 upgrade scenario. With the imminent increase on luminosity to $5 \times 10^{34} \mathrm{~cm}^{-2} \mathrm{~s}^{-1}$ and center of mass collision energy of $14 \mathrm{TeV}$ an unprecedented and hostile radiation environment will be created, the most affected detectors will be the ones located in the forward region where the intense flux of neutrons and photons could potentially degrade the detector performance. Using FLUKA simulation the expected radiation environment is estimated for the regions of interest, possible shielding scenarios are proposed and the effect on the detector performance is discussed.

Technology and Instrumentation in Particle Physics 2014,

2-6 June, 2014

Amsterdam, the Netherlands

\footnotetext{
* Speaker.

${ }^{\dagger}$ The corresponding author is supported by the Qatar National Research Fund (QNRF) under project NPRP-5-4641-080.
} 


\section{Introduction}

The Large Hadron Collider [1] (LHC) culminated its very successful first data taking period with the announcement of the discovery of a new particle with a mass of $125 \mathrm{GeV}$ and with properties consistent with the predicted Higgs boson particle [2], in order to measure with high precision the properties of this new particle and to continue the search for new physics phenomena the LHC experiments have started a series of detector upgrades and maintenance to restart activities with a substantial increase on the center of mass collision energy of $14 \mathrm{TeV}$ and eventually reach an optimal luminosity of $5 \times 10^{34} \mathrm{~cm}^{-2} \mathrm{~s}^{-1}$. Such a big increase in the number of collisions per second comes with an unavoidable and unprecedented radiation environment. The most affected sub-systems will be the ones located in the forward region (or end-caps) where the particle flux is more intense. The expected radiation environment can be studied using Monte Carlo simulation, nevertheless the current CMS reconstruction and simulation software (based on GEANT4) is not optimized to properly include the contribution of the neutron induced backgrounds due to limitations in the simulation time (current maximum value of 500ns while neutrons can have a time of flight of the order of seconds). This restriction is not present in the FLUKA [3] simulation software offering the possibility to study the radiation environment at the CMS experiment.

\section{The CMS muon system}

The CMS [4] is one of the two multi-purpose experiments at the LHC, it was initially designed for the accurate identification, trajectory reconstruction and energy measurement of particles produced as a result of the proton-proton collisions. Many processes both in Standard Model and new physics searches (including the Higgs boson) contain events with muons in the final state, therefore an excellent reconstruction efficiency and resolution is demanded. The current CMS muon system [5] relies on three gaseous detector technologies, in the central region Drif Tubes (DT) and Resistive Plate Chambers (RPC) while in the forward (end-cap) region a combination of Cathode Strip Chambers (CSC) and RPCs is used. For the very forward region $(|\eta|>1.6)$ the muon identification relies only on CSC stations which performance was acceptable for the low luminosity data taking but during the high luminosity conditions the detector operation could be compromised due to the intense particle flux.

\section{The Phase-2 CMS muon system upgrade}

The CMS collaboration is planning the installation of new muon stations in the end-cap region in order to improve muon resolution, reduce the trigger rate and assure a high muon reconstruction efficiency. For the Phase-2 muon upgrade (expected to be completed by 2023) three new stations based on Gas Electron Multiplier [6] (GEM) technology will be installed: The so-called ME0 station will be the closest one to the interaction point and it will extend the muon detection coverage up to a value of $|\eta|=3$ or 4 depending on the outcome of the performance studies. The second and third GEM stations GE1/1 and GE2/1 will be installed just in front of the existing ME1/1 and ME2/1 CSC stations respectively to act as a combine muon detection system and improve the muon resolution and triggering. Additionally two new RPC stations RE3/1 and RE4/1 will be installed 
nearby the existing ME3/1 and ME4/1 CSC stations respectively. GEM detector technology was chosen due to its excellent spacial resolution [7] and high rate capabilities (up to few $\mathrm{kHz}$ ). The proposed position of these new muon stations can be visualized in figure 1 .

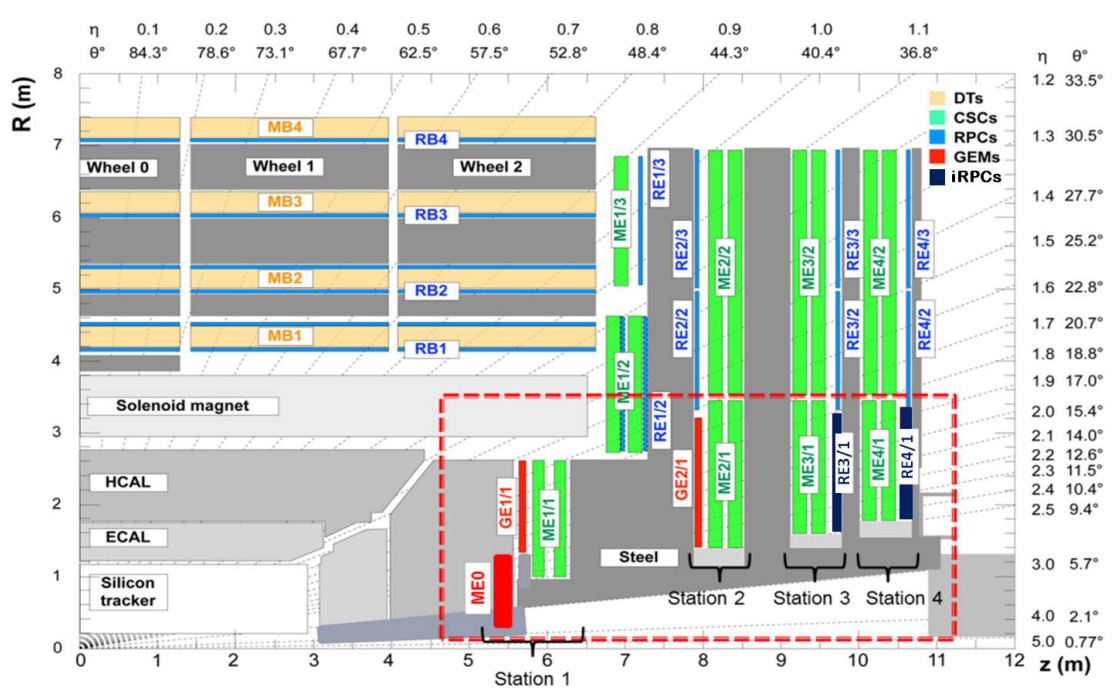

Figure 1: CMS phase-2 muon upgrade scenario: Three new stations based on Gas Electron Multiplier (GEM) technology and two new RPC stations will be installed to enhance the muon detection capabilities in the forward region, improve muon resolution and control the trigger rates during the high luminosity LHC scenario.

\section{Radiation Environment at CMS}

Except for the small contribution from machine related backgrounds the radiation field [8] is determined by the minimum bias events, which usually do not contain any interesting physics signatures. Given the fact that there is no available collision data at $14 \mathrm{TeV}$ the proton-proton cross section and the structure of minimum bias events is estimated using extrapolations from experimental data at lower energy. In the cascade propagation Hadronic and Electromagnetic showers are dissipated by the interaction in the different materials of the detectors, the remaining particles are mostly neutrons which are attenuated only by nuclear scattering processes and can therefore travel substantial distances. When a neutron has experienced numerous elastic scatterings, it will slow down to a limit, where its average momentum transfer in all subsequent collision is zero, most neutrons are slowed down to thermal energies before being absorbed by a nucleus. Neutron capture reactions may constitute a problem since the new isotope is usually formed in an excited state. Although the neutron energy may have been less than $1 \mathrm{eV}$ the resulting nuclear excitation can amount to several MeV. This excess energy is released in formed of capture gammas. Furthermore if the photons interact by Compton scattering electrons and positrons with energies of the order of $\mathrm{MeV}$ can ionize the detector sensitive material and create "fake" signals.

\section{FLUKA simulation}

FLUKA is a general purpose tool for calculation of particle transport and interactions with 
matter, covering a wide range of application including experimental high energy physics. The FLUKA simulation framework can handle complex geometries such as the CMS detector by using an improved version of the well-known Combinatorial Geometry (CG) package. The FLUKA simulation has evolved during the last years comparing simulation results against experimental data showing good agreement. The simulation requires the best possible description of the detector geometry, the beam particle type (in this case protons), the energy per beam, the particle energy thresholds and the output scoring options which in the case of radiation studies the most important quantity is the particle flux expressed as number of particles per seconds per area $\left(\mathrm{Hz} / \mathrm{cm}^{2}\right)$.

\section{Simulation Input}

A phase-2 CMS geometry scenario was built for FLUKA simulation based on the best knowledge of the detector at that time (as shown in figure 2). It includes improvements to the beam-pipe description and to the muon chamber shielding with respect to previous version used for the first data taking (Run-I). In this phase-2 model the end-cap calorimeters description is based on a preliminary model of one of the proposed calorimetry scenarios for Phase-2 named as High Granularity Calorimeter (HGC) option and the HE rebuild with a coverage up to $|\eta|=3$ in the pseudo-rapidity range. Additional shielding material based on borated-polyethylene and lead-antimony materials was used to cover the ME0 installation region to further reduce the neutron and photon fluxes. Primary proton-proton collisions with an energy of $7 \mathrm{TeV}$ per beam were used. Inelastic collision cross section used for normalization is $80 \mathrm{mb}$. Used simulation cut offs are: Hadrons $1 \mathrm{keV}$, Neutrons $0.01 \mathrm{meV}$, Photons $3 \mathrm{keV}$, Electrons $30 \mathrm{keV}$. Photons and Electrons have significantly higher cut-offs in some regions.

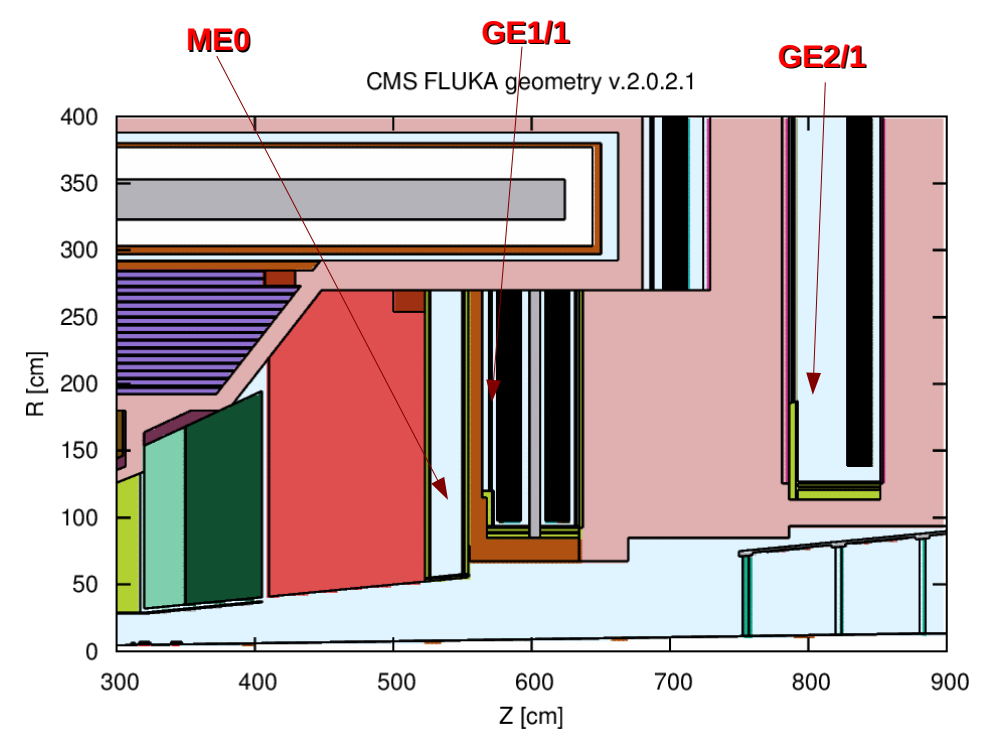

Figure 2: CMS FLUKA geometry for Phase-2 upgrade scenario it includes improvements in the beam-pipe and muon chamber shielding description compared with previous version. The end-cap calorimeters are based on a preliminary model of the High Granularity Calorimeter and the HE rebuild. Empty space is allowed for the computation of the radiation environment in the ME0, GE1/1 and GE2/1 region. 

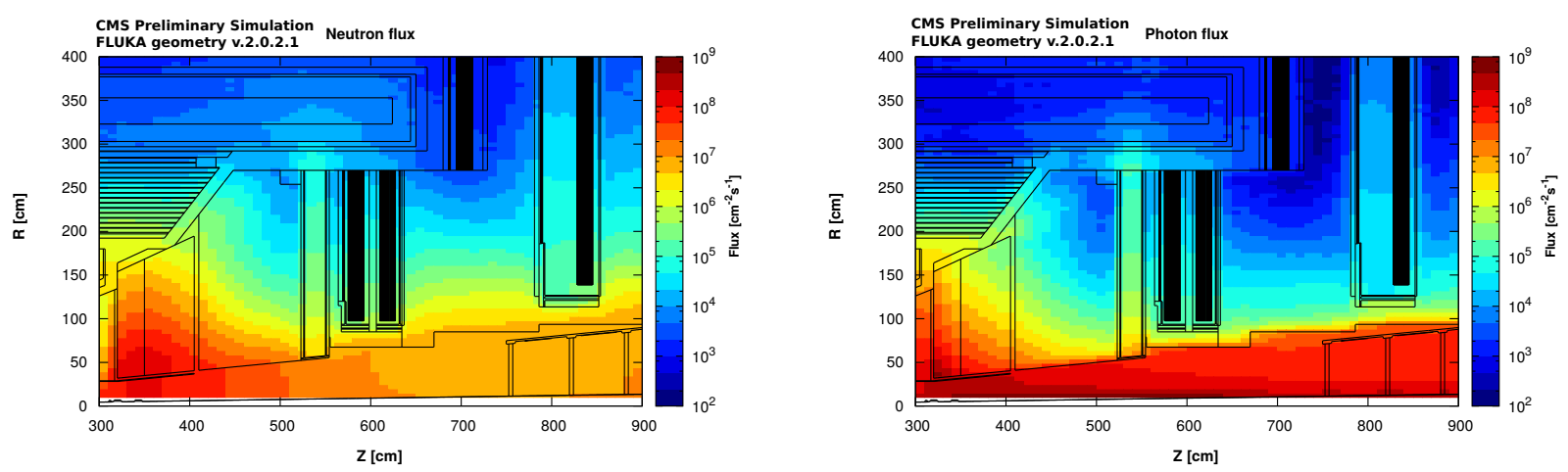

Figure 3: Monte Carlo estimation of the neutron (left) and photon (right) fluxes in the CMS forward region covering the end-cap Calorimeter and the first muon stations including the existing ME1/1 and ME2/1 CSC stations and the future location of the new GEM detector stations (ME0, GE1/1 and GE2/1).

\section{Results}

The particle flux was obtained for the CMS forward region using FLUKA simulation and with the initial parameters described in section 6. The CMS detector region defined in the simulation covers the proposed location of the new GEM stations and also the end-cap calorimeter which material composition has strong influence on the particle flux reaching the muon stations. The two dimensional plots presented in figure 3 show the particle flux in the $R, z$ space, where $R$ is the CMS radial coordinate and $z$ is the distance respect to the interaction point (pp collision), the flux value is color coded and showing the most intense contribution near the beam-pipe location. The plot in figure 4 shows the particle flux projection in the R coordinate for the three GEM stations and the different kind of particles, from this plot it is clear that the ME0 detector will receive the most intense particle flux with a maximum value of the order of $10^{7} \mathrm{~Hz} / \mathrm{cm}^{2}$ with the assumed luminosity. It is important to notice that the actual effect on the detector performance will be evaluated once the particle flux is combined with the detector sensitivity (probability of a particle to interact with the sensitive material and create a signal) which is a energy dependent variable and different for each kind of particle. Detector sensitivities are commonly studied using dedicated simulation (i.e. GEANT4) and taking into account the proper detector geometry description (cover, sensitive material, readout, etc.). Once the detector sensitivity factor is taken into account the particle flux may be reduced by few order of magnitudes to a value within the detector rate capabilities.

\section{Conclusions}

The radiation environment for the Phase-2 CMS muon upgrade was estimated using FLUKA simulation, assuming an instantaneous luminosity of $5 \times 10^{34} \mathrm{~cm}^{-2} \mathrm{~s}^{-1}$ and a center of mass collision energy of $14 \mathrm{TeV}$. The new muon stations based on GEM technology will be the most affected due to the position respect to the interaction point and beam-line. A baseline shielding was proposed to cover the ME0 volume and reduce the neutron and photon fluxes. According the simulation results a maximum particle flux of $10^{7} \mathrm{~Hz} / \mathrm{cm}^{2}$ is expected for ME0 stations, nevertheless after detector sensitivity factors this particle flux may be reduced few orders of magnitude to a value affordable by the GEM detector rate capabilities. 


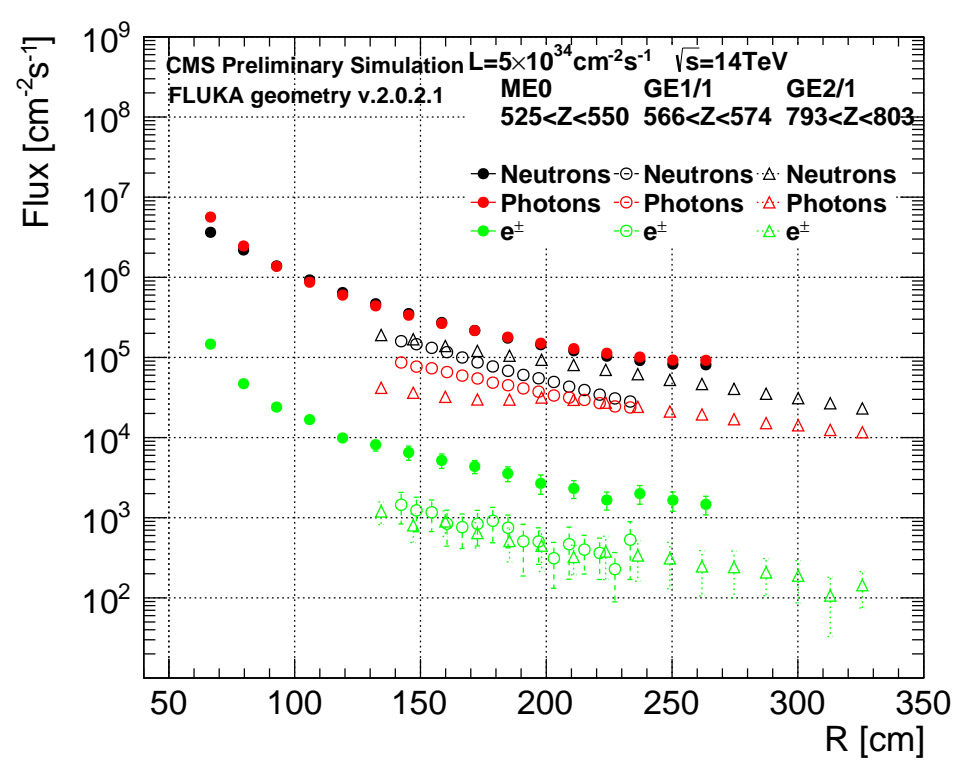

Figure 4: Particle flux as a function of the CMS radial coordinate for the three new GEM station and the different particle composition.

\section{Acknowledgements}

The corresponding author would like to thank the contribution of the radiation simulation group of the CMS Beam Radiation Instrumentation and Luminosity Project (BRIL) for their technical advice and geometry description files used in the simulation, the HPC (and/or scientific visualization) resources and services provided by the IT Research Computing group in Texas A\&M University at Qatar. The corresponding author is supported by the Qatar National Research Fund (QNRF) under project NPRP-5-464-1-080

\section{References}

[1] LHC Machine, Lyndon Evans and Philip Bryant 2008 JINST 3 S08001 doi:10.1088/1748-0221/3/08/S08001

[2] Observation of a new boson at a mass of $125 \mathrm{GeV}$ with the CMS experiment at the LHC, the CMS collaboration, Physics Letters B, Volume 716, Issue 1, 17 September 2012, Pages 30-31, ISSN 0370-2693, http://dx.doi.org/10.1016/j.physletb.2012.08.021

[3] "FLUKA: a multi-particle transport code". A. Ferrari, P.R. Sala, A. Fasso', and J. Ranft,CERN-2005-10 (2005), INFN/TC_05/11, SLAC-R-773

[4] The CMS experiment at the CERN LHC - CMS Collaboration (Chatrchyan, S. et al.) JINST 3 (2008) S08004

[5] The performance of the CMS muon detector in proton-proton collisions at $\sqrt{s}=7 \mathrm{TeV}$ at the LHC,The CMS collaboration 2013 JINST 8 P11002 
[6] Characterization of GEM Detectors for Application in the CMS Muon Detection System - Abbaneo, D. et al. IEEE Nucl.Sci.Symp.Conf.Rec. 2010 (2010) 1416-1422 arXiv:1012.3675 [physics.ins-det] RD51-NOTE-2010-005

[7] Development and Performance of Large Scale Triple GEM for CMS, D Abbaneo et al 2013 JINST 8 C11017 doi:10.1088/1748-0221/8/11/C11017

[8] The Radiation environment at the CMS experiment at the LHC, M. Huhtinen (CERN), http://inspirehep.net/record/421028/ 